\title{
Visualization of dynamic parameters of a multivariable system using Self-Organizing Maps.
}

\author{
J.J. Fuertes* M.A. Prada* M. Domínguez* P. Reguera* \\ I. Díaz ${ }^{* *}$ A.B. Díez ${ }^{* *}$ \\ * Instituto de Automática y Fabricación, Universidad de León, Escuela \\ de Ingenierías - Campus Universitario de Vegazana, León, 24071, \\ Spain.(e-mails: jjfuem@unileon.es, mapram@unileon.es, \\ mdomg@unileon.es,prega@unileon.es). \\ ** Departamento de Ingeniería Eléctrica, Electrónica de Computadores \\ y Sistemas, Universidad de Oviedo, Edificio Departamental 2 - \\ Campus de Viesques, Gijón, 33204, Spain. (e-mails: \\ idiaz@isa.uniovi.es, alberto@isa.uniovi.es).
}

\begin{abstract}
Self-organizing maps are excellent tools for information visualization. In this paper, they are used to extract information about the dynamic behavior of a multivariable system in several operating points. The input space of the SOM is constituted by extended vectors composed of the state of the process and the coefficients of the transfer functions that represent the dynamic behavior of the system for each operating point. New tools of visualization, such as maps of multivariable zeros or maps of relative gain, have been defined in order to explore the system so that suitable control strategies can be selected. The proposed method has been tested on a real multivariable system (a Quadruple-tank industrial scale model).
\end{abstract}

Keywords: Self-organizing map; Visual information; Multivariable systems: Dynamics; Transfer function; Scale systems

\section{INTRODUCTION}

Dimension reduction techniques (Kourti and MacGregor, 1995) are useful to map the complex input space of industrial processes onto a low output space that is easier to manage. These algorithms are often used along with visualization techniques, giving rise to visual data mining (Keim, 2002). In contrast to automated approaches, the aim of these techniques is to transform the information, without a significant loss, so that it can be projected onto visualizable low-dimensial spaces. Thus, it is possible to take advantage of the human ability to detect patterns and reason with graphical information.

A suitable algorithm for dimension reduction purposes is the Self-Organizing Map (SOM)(Kohonen, 1989, 2001). SOM has been successfully used as a tool for information visualization (Vesanto, 1999). Self-organizing maps have also been applied for temporal sequence processing and extraction of dynamic features (Barreto, 2007) in different areas such as prediction, speech recognition or control.

Multivariable processes require a comprehensive exploratory analysis to be understood in depth and recognize restrictions that lead to performance limitations in control

\footnotetext{
* This work was supported by the Spanish Ministerio de Educación y Ciencia under grant DPI2006-13477-C02-02. M. A. Prada was supported by a grant from the Consejería de Educación de la Junta de Castilla y León and the European Social Fund.
}

systems (Åstrom, 2000). These limitations are related to nonlinear zero dynamics (Zames, 1981). For that reason, it would be useful that the proposed techniques could be used not only to represent functional relationships among variables, but also to represent the dynamic behavior of proceses.

In this work, we use maps of dynamics (Díaz et al., 2008), a method based on SOM to represent dynamics of nonlinear or non-stationary industrial processes for multiple input-multiple output systems. The maps of dynamics are consistent with the component planes. They can be used together to analyze the relationships between the static variables and the dynamic behavior of the process. New visualization maps are defined to provide information about the zeros and the relative gain of the processes. Those tools are tested against a multivariable laboratory process (Johansson, 2000) with direct physical interpretation.

This paper is organized as follows. In section 2, selforganizing maps are reviewed. In section 3, our proposal of maps of dynamics based on SOM for multivariable proceses is presented. Section 4 describes the QuadrupleTank process, which constitutes the testing ground of the proposed approach. The results of these tests are discussed in section 5. Finally, conclusions and further work are described in section 6 . 


\section{SELF-ORGANIZING MAPS}

The SOM is an unsupervised neural network, based on competitive learning, that implements a nonlinear smooth mapping of a high-dimensional input space onto a lowdimensional output space. The neurons of the SOM form a topologically ordered low-dimensional lattice (usually twodimensional) that is an outstanding visualization tool to extract knowledge about the nature of the input space data.

Each neuron of the lattice is described by a $d$-dimensional prototype (codebook) vector $\mathbf{m}_{i}$, in the input space, and a position $\mathbf{g}_{i}$, in the low-dimensional grid of the output space. Neurons are related to the adjacent ones according to a neighborhood function $h_{i j}$, which works as a shortcut to allow lateral interactions.

The SOM algorithm implements its mapping in two stages. First, the best matching unit (BMU) of the input vector, $\mathbf{m}_{c}$, is selected by means of a competitive process $c=$ $\arg \min _{i}\left\|\mathbf{x}-\mathbf{m}_{i}(t)\right\|, i=1,2, \ldots, N$, where $\left\|^{\circ}\right\|$ denotes the Euclidean norm. Then, a cooperative step is performed, where the winning unit and its neighbors are adapted:

$$
\mathbf{m}_{i}(t+1)=\mathbf{m}_{i}(t)+\alpha(t) h_{c i}(t)\left[\mathbf{x}(t)-\mathbf{m}_{i}(t)\right]
$$

where $\alpha(t)$ is the learning rate and the neighborhood function $h_{c i}(t)$ is usually implemented as Gaussian. The success of the mapping critically depends on the parameters of this adaptive rule, which, in general, should decrease with time.

The SOM provides a good approximation to the input space (Luttrell, 1989), similar to vector quantization (VQ), and divides the space in a finite collection of Voronoi regions. It reflects the probability density function, since regions which a high probability of occurrence are magnified to gather a larger quantity of neurons.

The learning rule of the map drives the neighboring units, along with the BMU, towards the current input like a flexible net folding onto the input data sets. For that reason, a spatially-ordered and topology-preserving map emerges providing a faithful representation of the important features of the input.

Some useful representations such as the U-Matrix (Ultsch and Siemon, 1990) or the component planes (Tryba et al., 1989) have been developed. In fact, any scalar property of the input space can be visualized in the output space using the SOM. These maps associate the property value of a certain neuron, $\mathbf{m}_{i}$, to the corresponding coordinates of the lattice, $\mathbf{g}_{i}$. The property values are usually shown using a color level, so the $\mathbf{g}_{i}$ nodes form a picture where the property distribution for the process states is depicted.

As well as the SOM can be used to represent process variables, revealing the static relationships, it can also be used for an ordered representation of the different local dynamic behaviors of the process. These dynamical models are defined by vectors of parameters. Different approaches have been used, like time embedding using delays (Principe et al., 1998; Cho et al., 2006), analysis of the trajectory projected by the successive BMUs of the process (Srinivasa and Ahuja, 1999) or modifications in the activation or learning rules (Kangas, 1990; Euliano and Principe, 1999). Most of the approaches that use visualization to explore dynamic features are based on trajectories (Simula and
Kangas, 1995; Domínguez et al., 2007; Fuertes et al., 2007), but only a few of them have been proposed for explicit visualization of dynamical parameters (Díaz et al., 2008).

\section{MAPS OF DYNAMICS FOR MULTIVARIABLE SYSTEMS}

In this section, we present a method to analyze the dynamic behavior of multivariable linearized systems using Self-organizing Maps. Using the approach presented in Díaz et al. (2008), the input space of the SOM is augmented. The extended vectors are composed of a set of process variables and a set $\mathbf{p}_{i}$ of parameters of a parametric dynamical model

$$
y(k)=f\left(\phi(k), \mathbf{p}_{i}\right)
$$

where $\phi(k)$ is a vector of known data at sample $k$ and the choice of $f(\cdot, \cdot)$ defines the type of parametric model considered.

After a regular training, a SOM mapping between the parameter space and the output space will be set. Each unit will represent a local dynamic model, whose working point is defined by $\mathbf{x}$ and whose parameters are defined by $\mathbf{p}$. The resulting mapping is a consistent joint representation of the working point and its dynamics. Therefore, it can be compared with static maps such as the component planes to find links among them.

In this paper, codification of dynamics is done using a matrix of transfer functions that associates the multivariable outputs with the inputs. This matrix is derived from the linearization of the state-space equations of the system around several stationary operating points.

Let us consider a multivariable system characterized by a matrix of transfer functions $\mathbf{G}$, which relates the output $\mathbf{Y}$ with the input $\mathbf{U}$ :

$$
\left(\begin{array}{c}
y_{1} \\
y_{2} \\
\vdots \\
y_{i}
\end{array}\right)=\left(\begin{array}{cccc}
G_{11}(s) & G_{12}(s) & \cdots & G_{1 j}(s) \\
G_{21}(s) & G_{22}(s) & \cdots & G_{2 j}(s) \\
\vdots & \vdots & \ddots & \vdots \\
G_{i 1}(s) & G_{i 2}(s) & \cdots & G_{i j}(s)
\end{array}\right) \cdot\left(\begin{array}{c}
u_{1} \\
u_{2} \\
\vdots \\
u_{j}
\end{array}\right)
$$

where $G_{i j}$ is the transfer function that relates the output $i$ with the input $j$, as follows

$$
G_{i j}(s)=\frac{B_{i j}(s)}{A_{i j}(s)}
$$

where

and

$$
B_{i j}(s)=b_{0}^{i j}+b_{1}^{i j} s+\ldots+b_{n}^{i j} s^{n}
$$

$$
A_{i j}(s)=1+a_{0}^{i j} s+\ldots+a_{m-1}^{i j} s^{m} .
$$

The extended vectors conforming the input space of the SOM are defined as

$$
\mathbf{q}=\left[\mathbf{x}^{T}, \mathbf{p}^{T}\right]
$$

where

$$
\mathbf{x}^{T}=\left[x_{1}, \ldots, x_{i}\right]
$$

define the operating point of the process and

$$
\begin{array}{r}
\mathbf{p}^{T}=\left[b_{0}^{11}, b_{1}^{11}, \ldots, b_{n}^{11}, a_{0}^{11}, \ldots, a_{m-1}^{11}, \ldots\right. \\
\left.b_{0}^{i j}, b_{1}^{i j}, \ldots, b_{n}^{i j}, 1, a_{0}^{i j}, \ldots, a_{m-1}^{i j}\right]
\end{array}
$$

the coefficients of the transfer functions in that point.

As stated before, any scalar property of the input space can be represented. In our case, these properties provide 

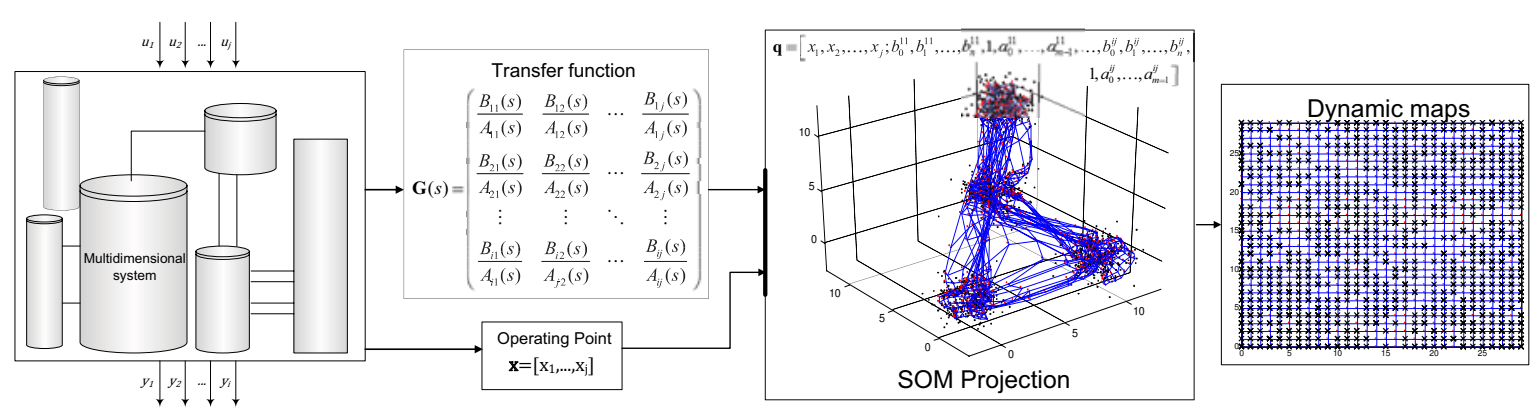

Fig. 1. Generation of maps of dynamics of multidimensional systems by means of the projection of parameter space.

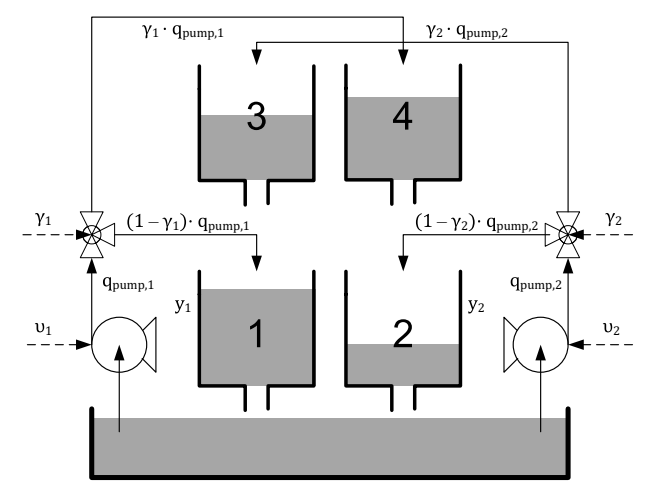

Fig. 2. Schematic diagram of the quadruple-tank process.

information about the dynamic behavior of the process. Figure 1 shows this idea graphically. A straightforward representation of the process dynamics are the component planes of model parameters. They describe, in an ordered fashion, the distribution of the model parameters for the different states reached by the process. The frequency response maps describe the variation of the frequency response of the process at frequency $\theta$ for each of the process states. The peak gain maps show the peak gains (resonance) of the process dynamics for every working point between two frequencies $\theta_{1}$ y $\theta_{2}$. Those maps were used (Díaz et al., 2008) to extract knowledge about the dynamic behavior of single input-single output linear systems (SISO). In this paper, a similar method is proposed to work with Multiple Input-Multiple Output systems (MIMO).

In addition to the previous representations, two new maps are proposed:

- Maps of multivariable zeros: The property shown in these maps is the multivariable zero of the parametric model associated to each neuron. The visualization of these maps reveals the operation points where the system is nonminimum-phase, i.e., one of the zeros is in the right half-plane (RHP). This introduces an additional difficulty for the system control.

- Maps of relative gain: The scalar property associated to each neuron is an element of the Relative Gain Array (RGA). The RGA, $\Lambda$, introduced by Bristol (1966), shows how the inputs and outputs of the system are coupled. It is defined as:

$$
\mathbf{\Lambda}=\mathbf{G}(0) * \mathbf{G}^{-T}(0),
$$

where $*$ denotes the Schur product (element-byelement matrix multiplication).
Finally, other visualization maps (Díaz Blanco et al., 2005) like fuzzy maps, which represent the output of a fuzzy inference system in the lattice, and maps of models, which associate to each neuron a function of the input space variables (for example a linear combination), can be used. They can be specially useful to analyze the dynamic behavior when combined with the maps defined above.

\section{QUADRUPLE-TANK PROCESS}

The quadruple-tank process proposed by Johansson (2000) is a multivariable system with two inputs and two outputs. It is composed of four water tanks interconnected to each other in such a way that the exit of the water-drainage of the upper tanks ends at the lower ones. Flow is supplied to the tanks by two symmetrically placed pumps. A threeway valve for each pump distributes the flow between the upper and lower tanks.

The purpose of the process is to control the levels in the lower tanks: $h_{1}$ and $h_{2}$. The process inputs are the variables of the pumps $v_{1}$ and $v_{2}$. The positions of the three-way valves $\left(\gamma_{1}\right.$ and $\left.\gamma_{2}\right)$ determine the proportion of the output flow that goes into the upper tanks. Figure 2 shows a diagram of the multivariable system and Table 1 shows the main variables and constants of the quadrupletank process.

The matrix of transfer functions, which links the input with the output for the different operational points of the process, must be obtained in order to form the extended

\begin{tabular}{c|c|c}
\hline Variable & Units & Description \\
\hline \hline$h_{i}$ & $\mathrm{~cm}$ & water level in the tanks \\
$h_{i}^{0}$ & $\mathrm{~cm}$ & steady-state tanks \\
$x_{i}$ & $\mathrm{~cm}$ & deviations of water levels $x_{i}=h_{i}-h_{i}^{0}$ \\
$q_{i}$ & $\mathrm{~cm}^{3} / \mathrm{s}$ & flows of the pumps to tanks \\
$v_{j}$ & $0-100$ & ratio of the pumps \\
$v_{j}^{0}$ & $0-100$ & steady-state pumps \\
$u_{j}$ & $0-100$ & deviations of pumps $u_{i}=v_{j}-v_{j}^{0}$ \\
$q_{p u m p, j}$ & $\mathrm{~cm} / \mathrm{s}$ & total flows of the pumps \\
$\gamma_{j}$ & $0-1$ & ratio of the flows \\
\hline \hline Constant & $\mathrm{Units}$ & Description \\
\hline \hline$A_{i}$ & $\mathrm{~cm}^{2}$ & cross-section of the tanks \\
$a_{i}$ & $\mathrm{~cm}^{2}$ & cross-section of the lower outlets \\
$g$ & $\mathrm{~cm}^{2} / \mathrm{s}$ & acceleration due to gravity \\
$k_{j}$ & $\mathrm{~cm}^{3} / \mathrm{s}$ & pump flow constants \\
$k_{c}$ & $\mathrm{~cm}$ & lower tank constants \\
\hline \hline
\end{tabular}

Table 1. Variables and constants of the quadruple-tank process 
vectors. The SOM is trained with these vectors to generate the maps of dynamics.

The equations applicable for the tanks and the pumps are Bernoulli's law, $q_{\text {out }}=a_{i} \sqrt{2 g h_{i}}$, and mass balances, $A \dot{h}=q_{\text {in }}-q_{\text {out }}$.

The model of the whole system can be obtained putting them together:

$$
\begin{aligned}
& \frac{d h_{1}}{d t}=-\frac{a_{1}}{A_{1}} \sqrt{2 g h_{1}}+\frac{a_{3}}{A_{1}} \sqrt{2 g h_{3}}+\frac{\left(1-\gamma_{1}\right) k_{1}}{A_{1}} v_{1} \\
& \frac{d h_{2}}{d t}=-\frac{a_{2}}{A_{2}} \sqrt{2 g h_{2}}+\frac{a_{4}}{A_{2}} \sqrt{2 g h_{4}}+\frac{\left(1-\gamma_{2}\right) k_{2}}{A_{2}} v_{2} \\
& \frac{d h_{3}}{d t}=-\frac{a_{3}}{A_{3}} \sqrt{2 g h_{3}}+\frac{\gamma_{2} k_{2}}{A_{3}} v_{2} \\
& \frac{d h_{4}}{d t}=-\frac{a_{4}}{A_{4}} \sqrt{2 g h_{4}}+\frac{\gamma_{1} k_{1}}{A_{4}} v_{1} .
\end{aligned}
$$

If these differential equations are linearized around an operating point $v_{1}^{0}, v_{2}^{0}$ using the Taylor series expansion, it is possible to obtain the state-space representation:

$$
\begin{aligned}
& \frac{d x}{d t}=\left(\begin{array}{cccc}
-\frac{1}{T_{1}} & 0 & \frac{A_{3}}{A_{1} T_{3}} & 0 \\
0 & -\frac{1}{T_{2}} & 0 & \frac{A_{4}}{A_{2} T_{4}} \\
0 & 0 & -\frac{1}{T_{3}} & 0 \\
0 & 0 & 0 & -\frac{1}{T_{4}}
\end{array}\right) x \\
& +\left(\begin{array}{cc}
\frac{\left(1-\gamma_{1}\right) k_{1}}{A_{1}} & 0 \\
0 & \frac{\left(1-\gamma_{2}\right) k_{2}}{A_{2}} \\
0 & \frac{\gamma_{2} k_{2}}{A_{3}} \\
\frac{\gamma_{1} k_{1}}{A_{4}} & 0
\end{array}\right) u \\
& y=\left(\begin{array}{cccc}
k_{c} & 0 & 0 & 0 \\
0 & k_{c} & 0 & 0
\end{array}\right) x
\end{aligned}
$$

where the time constants are

$$
T_{i}=\frac{A_{i}}{a_{i}} \sqrt{\frac{2 h_{i}^{0}}{g}} \quad i=1, \cdots, 4
$$

The Laplace transform of (12) yields the transfer matrix of the quadruple-tank system.

$$
\mathbf{G}(s)=\left(\begin{array}{cc}
\frac{\left(1-\gamma_{1}\right) c_{1}}{1+s T_{1}} & \frac{\gamma_{2} c_{1}}{1+\left(T_{3}+T_{1}\right) s+T_{3} T_{1} s^{2}} \\
\frac{\gamma_{1} c_{2}}{1+\left(T_{4}+T_{2}\right) s+T_{4} T_{2} s^{2}} & \frac{\left(1-\gamma_{2}\right) c_{2}}{1+s T_{2}}
\end{array}\right)
$$

Therefore, the extended input vectors of the SOM are, according to (7), as follows:

$$
\begin{array}{r}
\mathbf{q}=\left[\gamma_{1}, \gamma_{2}, v_{1}, v_{2}, h_{1}, h_{2}, h_{3}, h_{4} ;\left(1-\gamma_{1}\right) c_{1}, T_{1}, 1, \gamma_{2} c_{1}, T_{3} T_{1},\right. \\
\left.\left(T_{3}+T_{1}\right), 1, \gamma_{1} c_{2}, T_{4} T_{2},\left(T_{4}+T_{2}\right), 1,\left(1-\gamma_{2}\right) c_{2}, T_{2}, 1\right]
\end{array}
$$

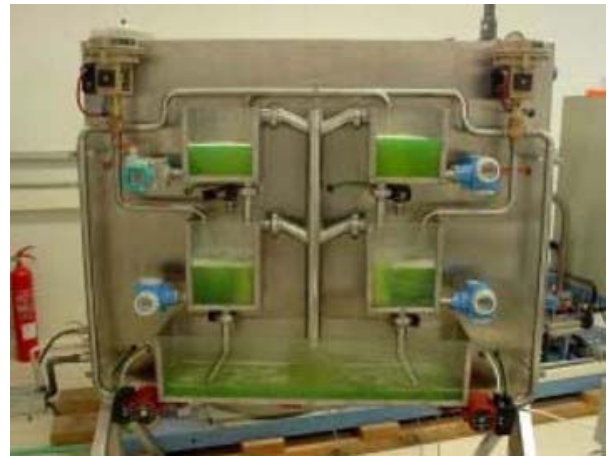

Fig. 3. Scale-model of the quadruple-tank process used to test the proposed method.

\section{EXPERIMENTAL RESULTS}

The proposed analysis, based on SOM, of the dynamic behavior of multivariable systems has been tested using a quadruple-tank industrial scale model. This equipment was developed by the group of Automatic Control of the University of León. Figure 3 shows a picture of the industrial scale model. It keeps the original structure of the quadruple-tank process proposed by (Johansson, 2000) but it is built using common industrial instrumentation:

- Grundfos UPE 25-40 flow pumps equipped with expansion modules Grudfos $M C$ 40/60 that control them by means of an analogical signal.

- Samson 3226-3760 three-way valves with positioners Samson 3760 to allow an external signal to control the opening degree of the valves.

- Endress\&Hauer PMC 731 pressure transmitters, for acquisition of the tank height.

- $S M C$ digital electrovalves to simulate perturbations on the level of the tank.

- Opto 22 SNAP Ultimate I/O data acquisition system, used as an interface between the applications and the scale model

The values of the descriptive parameters for this process, according to the model, are shown in Table 2.

The input space of the SOM is a 22-dimensional space formed by the state variables of the operating points of the process and the parameters of the transfer functions associated to those points, according to the expression (15), which defines the dynamic behavior in each point.

Approximately five thousand matrices of transfer functions were calculated, including all the possible operation modes of the system operation from the minimum to the maximum level of the tanks and from the minimum to the maximum opening of the valves.

A SOM with a $40 \times 40$ grid and a rectangular topology was trained using the batch training algorithm. The training length was 50 epochs. The neighborhood function was Gaussian $h_{i j}(t)=\exp \left(-\frac{d(i, j)}{\sigma(t)^{2}}\right)$ and $\sigma$ was made to

\begin{tabular}{c||c|c|c|c}
\hline Parameter & $A_{i}$ & $g$ & $a_{1}, a_{2}$ & $a_{3}, a_{4}$ \\
\hline \hline Value & $389.16 \mathrm{~cm}^{2}$ & $981 \mathrm{~cm}^{2} / \mathrm{s}$ & $2.5547 \mathrm{~cm}^{2}$ & $1.539 \mathrm{~cm}^{2}$ \\
\hline \hline
\end{tabular}

Table 2. Parameters of the scale model 


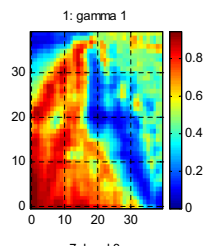

7: level3

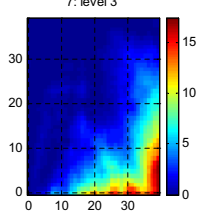

13: G12 a2

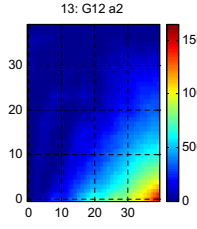

19: G21 a

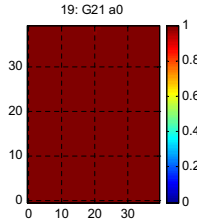

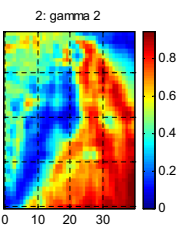

8: level 4

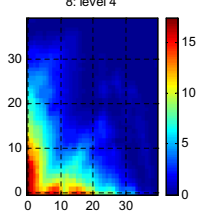

14: G12 a1

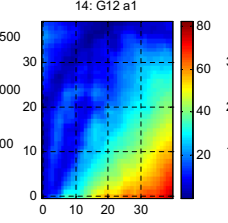

20: 622 bo

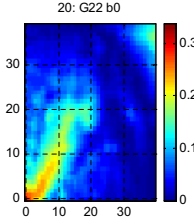

3: flow 1

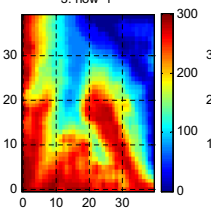

9: 611 bo

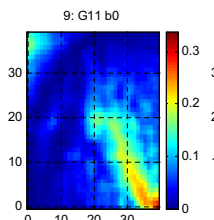

15: G21 a0

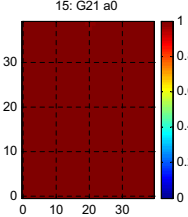

21: G22 a

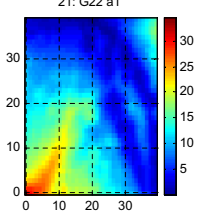

4: flow 2

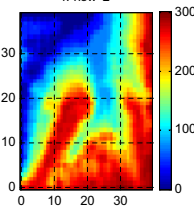

10: G11 a1

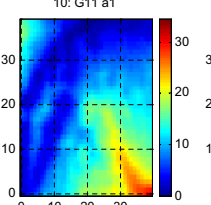

16: G21 bo

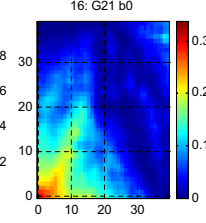

22: 622 a0

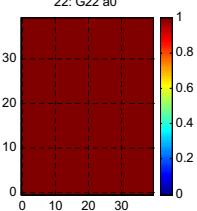

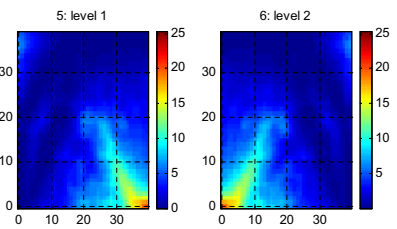

12: G12 bo

11: G11 a0

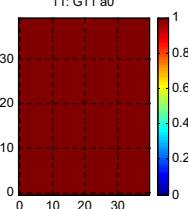

17: G21 a2
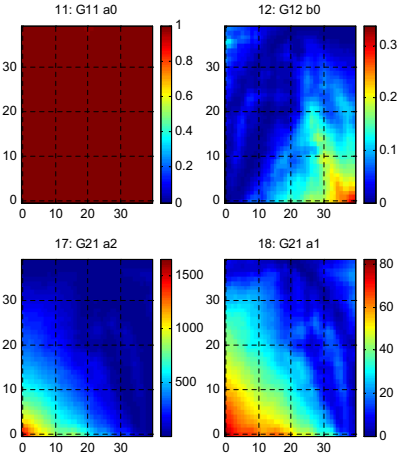

18: G21 a1

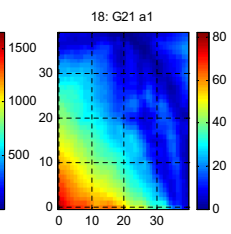

Fig. 4. Component planes of the process variables (the first 8) and the model parameters (the rest).

decrease monotonically from 10 to 1 . Figure 4 shows the component planes of that map.

The maps of multivariable zeros are shown in Fig. 5(a) and Fig. 5(b). Watching the first map, it is noticeable (value $<0)$ that the first zero of the system is always located in the negative semiplane. The area in the upper part of the maps is not considered in the analysis, since it corresponds with operation points in which the levels of the tanks are minimum and, therefore, the linear model is not valid. However, in the second figure, it can be distinguished a zone where the zero is positive and another one where the zero is negative. Therefore, the system clearly has two different operational zones (again, value greater or less than 0 in the map):

- The zone where the system is nonminimum phase, when one of the zeros is in the RHP (right-half plane). The nonminimum-phase system is harder to control if the influence of the coupled variables is rejected, so it is better to control the system with a centralized strategy instead of decentralized.

- The zone where the system is minimum-phase. Both zeros are in the LHP (left-half plane), so the system is simple to control. In this case, the coupling effect between variables is minimum and the system can be divided in simpler subsystems and therefore decentralized control is the suitable strategy.

This same conclusion is reached by analyzing the Relative Gain. The matrix of Relative Gain in the scale model, according to equation (10), is $\left(\begin{array}{cc}\lambda & 1-\lambda \\ 1-\lambda & \lambda\end{array}\right)$ where $\lambda=$ $\frac{\left(1-\gamma_{1}\right)\left(1-\gamma_{2}\right)}{1-\gamma_{1}-\gamma_{2}}$ The difficulty of the control depends on the value of $\lambda$, and it is specially hard for values of $\lambda<$ 0 according to McAvoy (McAvoy, 1983). Figure 5(c) shows the model map of the relative gain (evaluates the expression $\lambda>0$ ). In this map, the same two zones of operation are observed, corresponding to nonminimumand minimum-phase zones. It is even more clear than in the analysis of the maps of multivariable zeros. The blue zones, where $\lambda<0$, correspond with the zones of nonminimum-phase where the control becomes especially hard. In this case, the system has a zero in the positive semiplane (compare Fig. 5(b) and 5(c)).

Figure $5(\mathrm{~d})$ shows the model map $\gamma_{1}+\gamma_{2}$ for each stationary operating point of the process. It can be observed by comparing this map with the Fig. 5(c) that the zones where the system is nonminimum-phase (blue zones) correspond with those where $\gamma_{1}+\gamma_{2}$ is included in the interval [1,2] (strip of color from yellowish to reddish in Fig. 5(d)) When valves are in this interval, the sum of the flows to the upper tanks is greater than the flows to the lower ones, so it is more complicated to control the levels $y_{1}$ and $y_{2}$.

\section{CONCLUSION}

In this paper, we present a method, based on Selforganizing Maps, to extract information about the dynamic behavior of multivariable systems. Vectors extended with the coefficients of the transfer functions that relate inputs and outputs of the system for each operating point are used as the input space of the SOM. Once the SOM is trained, similar dynamic behaviors of the process are grouped in similar regions. This allows obtaining an ordered representation of the dynamic of the process. As the output space is low dimensional (usually $2 \mathrm{D}$ or $3 \mathrm{D}$ ), these regions can be visualized.

This visual character of the SOM allows us to define new visualization maps that help to discover dynamic properties of the process. Maps of multivariable zeros are very useful to classify the zones where the system is 


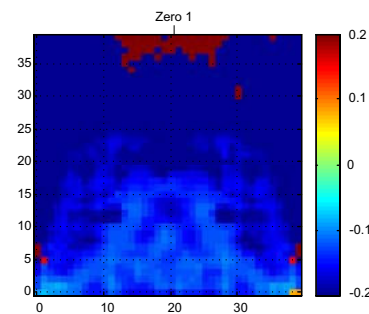

(a) Map of multivar. zeros 1

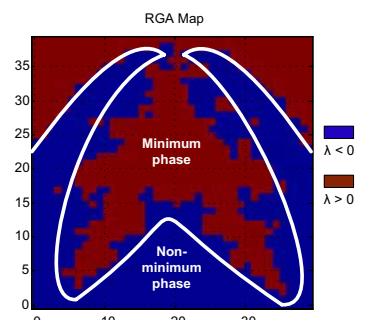

(c) Map of Relative gain $>0$

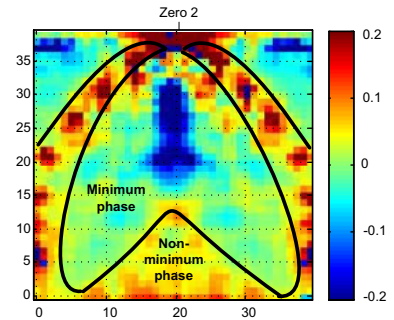

(b) Map of multivar. zeros 2

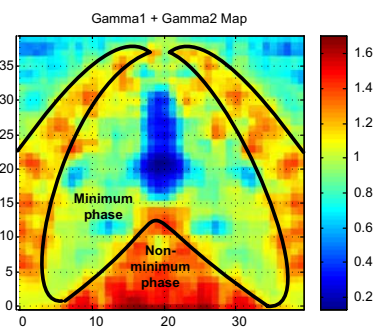

(d) Model map of $\gamma_{1}+\gamma_{2}$
Fig. 5. Model maps

nonminimum-phase, whereas maps of relative gain let us find out where the application of a decentralized control is effective or not. The proposed method has been validated against a quadruple-tank industrial scale model designed to make experiences of multivariable level control.

Results suggest further work in the development of other useful visualization tools of dynamic parameters. This method might also be merged with an algorithm for local dynamic modeling, like the ones cited in section 1 , to provide results directly from the process variables.

\section{REFERENCES}

Karl J. Åstrom. Limitations on control system performance. European Journal of Control, 6(1):2-20, 2000.

Guilherme A. Barreto. Time Series Prediction with the Self-organizing map: A Review. In Pascal Hitzler and Barbara Hammer, editors, Perspectives on NeuralSymbolic Integration. Spring-Verlag, 2007.

E. H. Bristol. On a new measure of interactions for multivariable process control. IEEE Transactions on Automatic Control, AC-11:133-134, 1966.

Jeongho Cho, Jose C. Principe, Deniz Erdogmus, and Mark A. Motter. Modeling and inverse controller design for an unmaned aerial vehicle based on the selforganizing map. IEEE Trans. Neural Networks, 17(2): 445-460, 2006.

Ignacio Díaz, Manuel Domínguez, Abel A. Cuadrado, and Juan José Fuertes. A new approach to exploratory analysis of system dynamics using SOM. Applications to industrial processes. Expert Systems with Applications., 34(4):2953-2965, May 2008.

Ignacio Díaz Blanco, Abel A. Cuadrado Vega, Alberto B. Díez González, and Guillermo Ojea. Modelado visual de procesos industriales [Visual modeling of industrial processes]. Revista Iberoamericana de Automática e Informática Industrial, 2(4):101-112, 2005.

Manuel Domínguez, Perfecto Reguera, Juan J. Fuertes, Ignacio Díaz, and Abel A. Cuadrado. Internet-based remote supervision of industrial processes using Self- organizing maps. Engineering Applications of Artificial Intelligence, 20(6):757-765, September 2007.

N. R. Euliano and J. C. Principe. A spatio-temporal memory based on SOMs with activity diffusion. In E. Oja and S. Kaski, editors, Kohonen Maps, pages 253266. Elsevier, Amsterdam, 1999.

Juan J. Fuertes, Miguel Angel Prada, Manuel Domínguez, Perfecto Reguera, Ignacio Díaz, and Abel A. Cuadrado. Modeling of Dynamics using Process State Projection on the Self Organizing Map. In International Conference on Artificial Neural Networks 200\%, Porto, Portugal, 2007.

Karl Henrik Johansson. The Quadruple-Tank Process: A Multivariable Laboratory Process with an Adjustable Zero. IEEE Transaction on Control Systems Technology, 8(3):456-465, 2000.

Jari Kangas. Time-Delayed Self-Organizing Maps. In Proceedings of the International Joint Conference on Neural Networks, pages 331-336, 1990.

Daniel A. Keim. Information visualization and visual data mining. IEEE Trans. Vis. Comput. Graph., 8(1):1-8, 2002.

Teuvo Kohonen. Self-Organizing Maps. Springer-Verlag New York, Inc., Secaucus, NJ, USA, 3rd edition, 2001. ISBN 3-5406-7921-9.

Teuvo Kohonen. Self-Organization and Associative Memory. Springer, Berlin, 3rd edition, 1989.

T. Kourti and J. F. MacGregor. Process analysis, monitoring and diagnosis, using multivariate projection methods. Chemometrics and Intelligent Laboratory Systems, (28):3-21, May 1995.

S. P. Luttrell. Self-organisation: a derivation from first principles of a class of learning algorithms. In International Joint Conference on Neural Networks, IJCNN, volume 2, pages 495-498, 1989.

T. J. McAvoy. Interaction Analysis: Principles and applications. Instrument Society of America, 1983.

J.C. Principe, L. Wang, and M.A. Motter. Local dynamic modeling with self-organizing maps and applications to nonlinear system identification and control. Proc. IEEE, 86(11):2240-2258, 1998.

Olli Simula and Jari Kangas. Process monitoring and visualisation using Self-Organizing Maps. In Neural Networks for Chemical Engineers, pages 377-390. Elsevier Science B.V., 1995.

Narayan Srinivasa and Narendra Ahuja. A Topological and Temporal Correlator Network for Spatiotemporal Pattern Learning, Recognition and Recall. IEEE Transactions on Neural Networks, 10(2):356-371, 1999.

Viktor Tryba, S. Metzen, and Karl Goser. Designing basic integrated circuits by self-organizing feature maps. In Neuro-Nîmes '89. Int. Workshop on Neural Networks and their Applications, pages 225-235, Nanterre, France, November 1989. ARC; SEE, EC2.

Alfred Ultsch and H. P. Siemon. Kohonen's Self Organizing Feature Maps for Exploratory Data Analysis. In INNC Paris 90, pages 305-308. U. Dortmund, 1990.

Juha Vesanto. SOM-based data visualization methods. Intelligent Data Analysis, 3(2):111-126, 1999.

G. Zames. Feedback and optimal sensitivity: Model reference transformation, multiplicative semi-norms and approximate inverses. IEEE Trans. on Automatic Control, 26:301-320, 1981. 